\title{
Allocution de bienvenue
}

\author{
Par O. JAAG, Suisse, président du GIROM
}

Mesdames, Messicurs,

Si nous voulons avoir un habitat salubre, nous devons observer certaines conditions essentielles dont les principales sont: protéger les eaux contre la pollution et assainir les eaux polluées en épurant les eaux usées urbaines et industrielles avant de les déverser dans les exutoires; éliminer de manière à les rendre inoffensifs les déchets solides - ordures ménagères, balayures, huiles usées, résidus industriels, cadavres d'animaux, déchets de boucherie, etc., ainsi que les boues d'épuration fraîches ou digérées; lutter contre la pollution de l'atmosphère, contre les bruits excessifs et toutes autres immissions. L'importance d'un service organisé de ramassage de tous les déchets produits dans une commune ou dars une région n'est en général vraiment comprise que lorsque pour une raison ou une autre - par exemple en cas de grève du personnel de voirie - les ordures s'amoncèlent dans les rues et constituent un véritable danger pour la santé publique.

L'élimination des déchets est un devoir qui existe depuis toujours, mais surtout à partir de l'époque où les hommes ont commencé à créer des agglomérations et des centres industriels. L'ordre et la propreté sont des principes qui ont été observés à des degrés plus ou moins grands au cours des siècles. Dans une période comme la nôtre, la protection de notre habitat est une obligation impérieuse et le problème des déchets devient de plus en plus difficile à résoudre. En effet, nous devenons de plus en plus exigeants en matière de confort et l'industrie produit en quantités toujours croissantes des objets d'usage courant de toutes formes et de toutes grandeurs - ustensiles de cuisine, appareils de radio et télévision, machines à laver, réfrigérateurs, automobiles, etc. etc. Cela augmente à l'extrême les quantités de déchets que les communes et les régions ont le devoir d'éliminer et de rendre inoffensifs de manière aussi économique que possible. L'évolution économique devenue si rapide après la seconde guerre mondiale, l'automation et l'accroissement accéléré de la population font dévaler sur nous une avalanche de déchets dont il est impossible de prévoir la fin.

Au cours de la dernière quinzaine d'années, le volume des ordures ménagères a augmenté de plus de $100 \%$, principalement du fait de la vogue des emballages. En Allemagne Fédérale, par exemple, on a produit en 1951 pour 1,3 milliards de DM (prix coûtant) d'emballages et en 1965 pour 8 milliards de DM; pour 1970, ce chiffre est évalué à 12-13 milliards de DM. La question des «emballages perdus» donne lieu à une certaine inquiétude en ce qui concerne l'élimination, car il s'agit là de com- 
posants qui compliquent énormément le traitement des déchets. Comme on le voit, il importe de réviser et modifier continuellement les méthodes de traitement et d'élimination des déchets pour les approprier aux exigences changeantes et aux possibilités du moment. Il faut pour cela que les autorités responsables s'assurent la collaboration de la science et de la technique et se tiennent au courant - par les moyens de l'information, de la documentation et de la discussion - des progrès réalisés dans ces domaines, afin d'être capables de choisir, parmi toutes les possibilités techniques qui leur sont offertes, la solution optimale pour chaque cas particulier.

La tâche des autorités est extrêmement complexe et variée, car elles doivent prendre des décisions importantes en ce qui concerne l'organisation du ramassage, du transport, du traitement, de la réutilisation et de l'élimination des déchets, ainsi que de l'élimination des produits irréductibles qui résultent du traitement.

En ce qui concerne le ramassage et le transport des déchets, les services municipaux de voirie ont trouvé des solutions satisfaisantes. Le problème est toutefois plus compliqué lorsqu'il s'agit de décider ce que l'on fera des déchets ramassés. Les possibilités qui se présentent sont les suivantes:

La décharge non contrôlée, malheureusement encore pratiquée dans beaucoup d'endroits, défigure et dégrade le paysage et empeste l'atmosphère. Il est généralement reconnu que c'est une méthode d'élimination qui n'est plus tolérable à notre époque.

Elle fait maintenant place à la décharge contrôlée - "controlled tipping» ou "sanitary landfill" - pratiquée surtout en Grande Bretagne, mais aussi en France et dans d'autres pays. C'est une méthode plus compliquée et plus coûteuse, car elle nécessite des machines spéciales et un personnel plus nombreux; par contre, elle exclut pratiquement toutes immissions et permet de récupérer des terrains devenus inutilisables.

Le compostage est une méthode originaire des Pays-Bas dont les habitants ont découvert le moyen de transformer, par traitement approprié et fermentation à grande échelle, les composants organiques des ordures domestiques et de certains déchets industriels en un produit servant à améliorer le sol et permettant de revaloriser des terres autrefois incultes. Le compost de gadoues est en outre et principalement utilisé par les néerlandais, en vertu de la propriété qu'il a de former de l'humus, pour rendre encore plus fertiles leurs terres agricoles.

Une voie analogue a été suivie en Allemagne Fédérale où l'on recherche activement de nouvelles utilisations pour le compost, notamment à l'Ecole de viticulture de Weinsberg.

On s'est rendu compte relativement tôt que les grandes villes, en particulier lorsqu'elles sont situées dans une région densément peuplée, ne peuvent pas avoir recours au compostage comme méthode d'élimination de leurs déchets urbains et industriels. C'est pourquoi de nombreuses villes du monde entier ont adopté une méthode plus radicale, à savoir l'incinération. Cette évolution est grandement favorisée par les fabricants de fours et de machines entre lesquels règne une très vive concurrence.

Les autorités qui se décident en faveur de l'incinération ont encore à choisir, suivant les circonstances locales et régionales, entre l'incinération avec récupération d'énergie et l'incinération sans récupération d'énergie; si elles prennent la première de ces deux possibilités, elles doivent encore décider si l'énergie récupérée sera utilisée pour la production de chaleur, de vapeur ou de courant électrique. 
Soyons heureux qu'il y ait tant de possibilités d'élimination, car l'agriculture ne suffirait jamais à absorber sous forme de compost la totalité des quantités immenses de déchets qui sont produites aujourd'hui. Notons cependant qu'il existe un très réel besoin en produits formateurs d'humus pour parer à l'appauvrissement du sol causé par les récoltes annuelles.

Nous voyons donc combien il est important de trouver la solution optimale pour chaque cas particulier. Cela implique une recherche intense dans divers secteurs, travaux dont les résultats doivent être communiqués aux milieux compétents et intéressés. C'est ce que nous nous proposons d'accomplir dans le présent congrès qui est le $4^{\mathrm{c}}$ Congrès du Groupe international de recherches sur les ordures ménagères.

Lorsqu'en 1955 la nécessité s'imposa en Suisse d'examiner sérieusement l'ensemble du problème, nous invitâmes un petit nombre des spécialistes européens les plus compétents à venir discuter avec nous, à l'Ecole Polytechnique Fédérale de Zurich, des diverses questions à l'ordre du jour. Le résultat le plus important de cette réunion fut la résolution qui fut prise de constituer le Groupe international de recherches sur les ordures ménagères, une organisation qui comprend aujourd'hui les groupes nationaux de l'Allemagne, de la France, de la Belgique, de l'Italie, d'Israël, de l'Autriche, des Pays-Bas et de la Suisse. Le GIROM commença immédiatement la publication d'un Bulletin d'information rédigé par le $D^{r}$ R. Braun, chef de la section d'études et de consultations sur les déchets solides de 1'EAWAG. Le Bulletin d'information, dont 35 numéros ont déjà paru, est édité en allemand, français, italien et anglais et diffusé dans environ 80 pays.

Grâce à cette organisation, pratiquement tous les spécialistes des questions relatives aux déchets solides peuvent collaborer sur le plan international; trois congrès internationaux du GIROM ont déjà eu lieu, à savoir à Scheveningen (Pays-Bas) en 1959, à Essen (République Fédérale d'Allemagne) en 1962 et à Trente (Italie) en 1965 .

Le Congrès de Trente nous a laissé un souvenir durable, mais la recherche et la pratique ont fait de grands progrès depuis 1965 dans les domaines du nettoiement des villes et du traitement des déchets solides, progrès dont il sera fait mention dans la première conférence principale.

Nous avons choisi une formule nouvelle pour notre $4^{\mathrm{e}}$ Congrès international dont nous espérons qu'il donnera de meilleurs résultats pratiques qu'un congrès conventionnel consistant presque uniquement en un certain nombre de conférences. Les discussions tiendront une place importante dans le présent congrès; six groupes de travail ont été formés dont chacun est dirigé par un éminent spécialiste qui nous a fait parvenir d'avance une conférence d'introduction sur le sujet attribué à son groupe particulier. Les six conférences d'introduction, ainsi que les co-conférences qui nous sont parvenues à temps, ont été envoyées à tous les participants quelque temps avant le congrès, afin de leur donner la possibilité de se préparer en vue de la discussion.

Avant que le congrès ne prenne fin, les chefs de groupe présenteront un rapport de synthèse des travaux de leurs groupes respectifs, rapports dont nous espérons qu'ils poseront les jalons pour les futurs travaux de recherche dans le domaine si vaste de l'élimination des déchets solides.

L'exposition PRO AQUA présente les produits des entreprises travaillant dans presque tous les secteurs de l'élimination des déchets solides. Dix excursions ont été 
organisées qui permettront aux participants de visiter les principales usines suisses de traitement et d'élimination.

Monsieur Pierre Clairgeon, Président de l'INTAPUC, avait très aimablement accepté de prononcer la seconde conférence principale dans le cadre de notre Congrès international. Les organisateurs du congrès attendaient avec plaisir l'exposé que cet éminent praticien devait faire sur "Les devoirs de l'Etat et des communes en ce qui concerne la conservation de notre habitat». Puis nous vint de Paris la foudroyante nouvelle que Monsieur Clairgeon venait de décéder le 18 novembre 1968 des suites d'un infarctus cardiaque, à l'âge de 66 ans. Nous eûmes peine à y croire, car, peu avant, nous l'avions rencontré pour préparer avec lui le congrès qui commence aujourd'hui et il donnait alors l'impression d'être un homme en excellente santé, plein de joie de vivre et en pleine activité. Notre douleur est grande, car nous avions tous beaucoup d'amitié et d'estime pour cet homme si aimable, si serviable et si actif. Il venait de prendre sa retraite de Directeur Général des Services techniques de la Ville de Paris. C'est lui qui avait organisé le programme général d'assainissement de la région parisienne et préparé la construction de la grande station d'épuration d'Achères. Le réseau de trafic express de Paris, des réservoirs d'eau potable et des bassins de protection contre les inondations sont les œuvres que Monsieur Clairgecn réalisa en sa qualité de Directeur technique de la Voirie parisienne; d'autres œuvres sont restées à l'état de projet dont la réalisation incombera à son successeur. En collaboration avec l'Electricité de France, Monsieur Pierre Clairgeon contribua pour une large part à la construction de l'usine d'incinération des gadoues d'Issy-les-Moulineaux, une des plus grandes usines de ce genre dans le monde. Sa participation à la transformation de Paris en grande ville mondiale fut très estimée par les autorités qui lui en ont rendu honneur.

C'est donc avec un très vif chagrin que nous avons dû dire adieu à Monsieur Pierre Clairgeon; son souvenir restera avec nous, car nous ne pourrons jamais oublier ses mérites, sa personnalité si élevée et son sourire si charmant.

Une notice commémorative sur la vie et l'œuvre de Monsieur Pierre Clairgeon, écrite par M. Drouin, a paru dans le Bulletin d'information $n^{\circ} 34$ du GIROM.

Plus de 700 participants se sont inscrits au $4^{\circ}$ Congrès du GIROM. Cela prouve l'importance de la recherche, de la technique et de l'organisation, ainsi que la nécessité de l'information dans ce domaine si vaste qui est celui de l'élimination des déchets solides.

Au nom des organisateurs du Congrès je remercie tous ceux qui témoignent un intérêt si vif pour les questions qui seront traitées ici. Je remercie aussi tout spécialement tous ceux et celles qui ont travaillé à la réussite de notre congrès, que ce soit en qualité d'orateur, de chef de discussion, de guide d'excursions ou dans les services administratifs, en mettant à notre disposition leur temps, leur science et leurs capacités.

Adresse de l'auteur: Prof. Dr. O. Jaag, directeur de l'Institut fédéral pour l'aménagement, l'épuration et la protection des eaux (EAWAG), à l'Ecole Polytechnique Fédérale de Zurich, Physikstrasse 5, CH-8044 Zurich (Suisse). 